\title{
Probing non-polar interstellar molecules through their protonated form: Detection of protonated cyanogen $\left(\mathrm{NCCNH}^{+}\right)^{\star}$
}

\author{
M. Agúndez ${ }^{1}$, J. Cernicharo ${ }^{1}$, P. de Vicente ${ }^{2}$, N. Marcelino ${ }^{3}$, E. Roueff ${ }^{4}$, A. Fuente ${ }^{5}$, M. Gerin ${ }^{6}$, M. Guélin ${ }^{7}$, C. Albo ${ }^{2}$, \\ A. Barcia², L. Barbas², R. Bolaño², F. Colomer², M. C. Diez², J. D. Gallego ${ }^{2}$, J. Gómez-González², \\ I. López-Fernández ${ }^{2}$, J. A. López-Fernández², J. A. López-Pérez ${ }^{2}$, I. Malo², J. M. Serna², and F. Tercero² \\ ${ }^{1}$ Instituto de Ciencia de Materiales de Madrid, CSIC, C/ Sor Juana Inés de la Cruz 3, 28049 Cantoblanco, Spain \\ e-mail: marcelino.agundez@icmm.csic.es \\ 2 Centro Nacional de Tecnologías Radioastronómicas y Aplicaciones Geoespaciales (CNTRAG), Observatorio de Yebes (IGN), Spain \\ 3 INAF, Istituto di Radioastronomia, via P. Gobetti 101, 40129 Bologna, Italy \\ ${ }_{5}^{4}$ LERMA, Observatoire de Paris, PSL Research University, CNRS, UMR 8112, place Janssen, 92190 Meudon Cedex, France \\ 5 Observatorio Astronómico Nacional (OAN), Calle Alfonso XII, No 3, 28014 Madrid, Spain \\ ${ }^{6}$ LERMA, Observatoire de Paris, École Normale Supérieure, PSL Research University, CNRS, UMR 8112, 75014 Paris, France \\ 7 Institut de Radioastronomie Millimétrique, 300 rue de la Piscine, 38406 St. Martin d'Héres, France
}

Received 1 June 2015 / Accepted 23 June 2015

\section{ABSTRACT}

\begin{abstract}
Cyanogen $(\mathrm{NCCN})$ is the simplest member of the series of dicyanopolyynes. It has been hypothesized that this family of molecules can be important constituents of interstellar and circumstellar media, although the lack of a permanent electric dipole moment prevents its detection through radioastronomical techniques. Here we present the first solid evidence of the presence of cyanogen in interstellar clouds by detection of its protonated form toward the cold dark clouds TMC-1 and L483. Protonated cyanogen $\left(\mathrm{NCCNH}^{+}\right)$has been identified through the $J=5-4$ and $J=10-9$ rotational transitions using the $40 \mathrm{~m}$ radiotelescope of Yebes and the IRAM $30 \mathrm{~m}$ telescope. We derive beam-averaged column densities for $\mathrm{NCCNH}^{+}$of $(8.6 \pm 4.4) \times 10^{10} \mathrm{~cm}^{-2}$ in TMC-1 and $(3.9 \pm 1.8) \times$ $10^{10} \mathrm{~cm}^{-2}$ in L483, which translate into fairly low fractional abundances relative to $\mathrm{H}_{2}$, in the range $(1-10) \times 10^{-12}$. The chemistry of protonated molecules in dark clouds is discussed, and it is found that, in general terms, the abundance ratio between the protonated and non-protonated forms of a molecule increases with increasing proton affinity. Our chemical model predicts an abundance ratio $\mathrm{NCCNH}^{+} / \mathrm{NCCN}$ of $\sim 10^{-4}$, which implies that the abundance of cyanogen in dark clouds could be as high as $(1-10) \times 10^{-8}$ relative to $\mathrm{H}_{2}$, i.e., comparable to that of other abundant nitriles such as $\mathrm{HCN}, \mathrm{HNC}$, and $\mathrm{HC}_{3} \mathrm{~N}$.
\end{abstract}

Key words. astrochemistry - line: identification - ISM: clouds - ISM: molecules - radio lines: ISM

\section{Introduction}

Nitriles, i.e., molecules containing a functional group $-\mathrm{C} \equiv \mathrm{N}$, are present in diverse astronomical environments. In particular, cyanopolyynes, $\mathrm{H}-(\mathrm{C} \equiv \mathrm{C})_{n}-\mathrm{C} \equiv \mathrm{N}$, are commonly found in cold interstellar clouds (Broten et al. 1978; Bell et al. 1997), in circumstellar envelopes around carbon-rich evolved stars (Winnewisser \& Walmsley 1978; Pardo et al. 2005), and in planetary atmospheres with a high content of carbon and nitrogen, such as that of Titan (Kunde et al. 1981; Coustenis et al. 1991).

It has been suggested that dicyanopolyynes, molecules that contain two cyano groups, $\mathrm{N} \equiv \mathrm{C}-(\mathrm{C} \equiv \mathrm{C})_{n}-\mathrm{C} \equiv \mathrm{N}$, could be abundant in interstellar and circumstellar clouds (Kołos \& Grabowski 2000; Petrie et al. 2003). However, these molecules cannot be detected through their rotational spectrum because they lack a permanent electric dipole moment. The simplest member of this series, cyanogen $(\mathrm{NCCN})$, is thought to be a major precursor of the $\mathrm{CN}$ radical observed in cometary comae (e.g., Fray et al. 2005), although detecting it in a comet still remains challenging.

* Based on observations carried out with the IRAM $30 \mathrm{~m}$ Telescope and the Yebes $40 \mathrm{~m}$ Telescope. IRAM is supported by INSU/CNRS (France), MPG (Germany) and IGN (Spain). The 40 m radiotelescope at Yebes Observatory is operated by the Spanish National Geographic Institute (IGN, Ministerio de Fomento).
Moreover, infrared observations carried out with Voyager 1 have identified NCCN in the atmosphere of Titan (Kunde et al. 1981), and the larger homologue $\mathrm{NC}_{4} \mathrm{~N}$ has long been thought to be present as well (Jolly et al. 2015). It is also worth noting that a chemical cousin of cyanogen in which one $\mathrm{N}$ atom is substituted by a $\mathrm{P}$ atom, NCCP, has been tentatively identified in the $\mathrm{C}$-rich envelope IRC +10216 (Agúndez et al. 2014).

Given the difficulty of directly detecting NCCN and larger dicyanopolyynes in cold interstellar clouds, it has been proposed that indirect strategies for probing their presence would be to observe chemically related molecules such as the polar metastable isomer $\mathrm{CNCN}$ or the protonated form $\mathrm{NCCNH}^{+}$ (Petrie et al. 2003). Here we report the first detection in the interstellar medium of $\mathrm{NCCNH}^{+}$, the protonated form of cyanogen, toward the cold dark clouds TMC-1 and L483.

\section{Observations}

Protonated cyanogen is a highly polar linear molecular cation. Its rotational spectrum has been characterized in the laboratory from microwave to millimeter wavelengths (Amano \& Scappini 1991; Gottlieb et al. 2000) and its electric dipole moment has been calculated as 6.448 Debye (Botschwina \& Sebald 1990). We have observed the $J=5-4$ transition at $44.4 \mathrm{GHz}$ with the 
Yebes $40 \mathrm{~m}$ telescope and the $J=10-9$ transition at $88.8 \mathrm{GHz}$ with the IRAM $30 \mathrm{~m}$ telescope toward the cold dark clouds TMC- 1 and L483 at the positions observed by Marcelino et al. (2005, 2007) and Agúndez et al. (2008), respectively.

\subsection{IRAM $30 \mathrm{~m}$}

The IRAM 30 m observations were carried out using the EMIR $3 \mathrm{~mm}$ receiver and the fast Fourier transform spectrometer with a spectral resolution of $50 \mathrm{kHz}$. The frequency-switching technique was used to optimize the telescope time. The half power beam width (HPBW) of the IRAM $30 \mathrm{~m}$ telescope at $88.8 \mathrm{GHz}$ is 27 '. 3 . The observations of TMC-1 are part of a spectral line survey at $3 \mathrm{~mm}$ (Marcelino et al. 2005, 2007, 2009). Most of the observations at $88.8 \mathrm{GHz}$ used in this article were taken in February 2012, when the good weather conditions resulted in a system temperature of $\sim 70 \mathrm{~K}$. More details are given in Cernicharo et al. (2012). The observations of L483 were taken from September to November 2014 during an observational campaign aimed at observing negative ions in dense molecular clouds and are described in detail in Agúndez et al. (2015).

\subsection{Yebes $40 \mathrm{~m}$}

The $40 \mathrm{~m}$ radiotelescope is located at Yebes (Guadalajara, Spain) at $980 \mathrm{~m}$ above sea level. Weather conditions are typically dry: the amount of precipitable water vapor ranges from $4 \mathrm{~mm}$ in winter to $14 \mathrm{~mm}$ in summer. The antenna has a homological design, and its optics is that of a Nasmyth radio telescope with several receivers in the Nasmyth cabin. The antenna is equipped with a cryogenic single-pixel dual-polarization $45 \mathrm{GHz}$ receiver built at Yebes, whose instantaneous bandwidth ranges between 41 and $49 \mathrm{GHz}$. The IF only processes bands of $500 \mathrm{MHz}$ and/or $1500 \mathrm{MHz}$ and its signal is injected into a fast Fourier transform spectrometer with several modules, which can be configured to provide 16384 channels on a bandwidth of $100 \mathrm{MHz}$ or $500 \mathrm{MHz}$. Observations reported in this article used two modules $100 \mathrm{MHz}$ wide with a spectral resolution of $6 \mathrm{kHz}$. Spectra were later smoothed to a spectral resolution of $12 \mathrm{kHz}$.

The observations toward TMC-1 and L483 were carried out in 9 and 12 periods, respectively, of 5-7 h during March and April 2015. System temperatures ranged between 120 and $200 \mathrm{~K}$. On-off observations were done with an integration time of $60 \mathrm{~s}$ and the off position located 600" away in right ascension. Calibration was repeated every $20 \mathrm{~min}$ and consisted of observing the sky and a hot load at ambient temperature. The sky opacity was estimated using the weather conditions measured $400 \mathrm{~m}$ away from the antenna and the atmospheric transmission model ATM (Cernicharo 1985; Pardo et al. 2001). We believe that the sky opacity has a maximum error of $10 \%$ (estimated by comparing with skydip measurements). To keep a good pointing and focus, continuum observations were performed every $20 \mathrm{~min}$ toward UOri (for TMC-1) and $\mathrm{OH} 26.5+0.6$ (for L483). The pointing and focus are estimated to be accurate within $5^{\prime \prime}$. The HPBW of the Yebes $40 \mathrm{~m}$ telescope at $44.4 \mathrm{GHz}$ is $42^{\prime \prime} .6$.

The final spectra of TMC- 1 and L483 were obtained by averaging 2440 and 2338 individual spectra, respectively, half of them with left(right) circular polarization. The intensity scale is given in antenna temperature $\left(T_{\mathrm{A}}^{*}\right)$, which corrects for the sky opacity and forward efficiency $(90 \%)$. The main beam efficiency has been estimated to be $36 \%$ in an elevation range between $20^{\circ}$ and $80^{\circ}$ from observations toward Venus and Saturn. Calibration can be considered correct within a $10 \%$ of uncertainty, mostly coming from the aperture efficiency and atmospheric conditions.
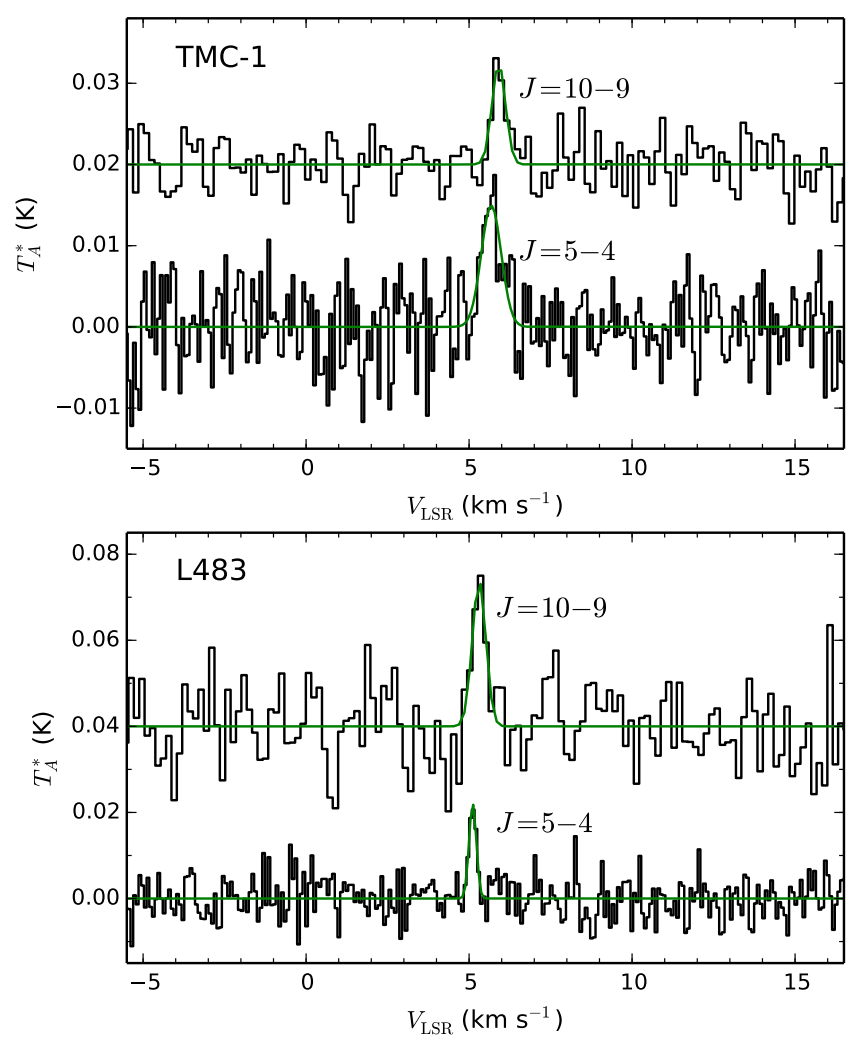

Fig. 1. Spectra of TMC-1 and L483 showing the emission lines assigned to the $J=5-4$ and $J=10-9$ rotational transitions of $\mathrm{NCCNH}^{+}$, lying at $44.4 \mathrm{GHz}$ and $88.8 \mathrm{GHz}$, respectively. The $T_{\mathrm{A}}^{*}$ rms of the spectra at $44.4 \mathrm{GHz}$ is $0.0045 \mathrm{~K}$ per $12 \mathrm{kHz}$ channel for both sources, while at $88.8 \mathrm{GHz}$ the $T_{\mathrm{A}}^{*} \mathrm{rms}$ is $0.0029 \mathrm{~K}$ and $0.0088 \mathrm{~K}$ per $50 \mathrm{kHz}$ channel, for TMC-1 and L483, respectively. The antenna temperature scale can be converted to main beam brightness temperature by dividing by $\left(B_{\text {eff }} / F_{\text {eff }}\right)$, which takes a value of $0.36 / 0.90$ for the Yebes $40 \mathrm{~m}$ telescope at $44.4 \mathrm{GHz}$ and of $0.82 / 0.90$ for the IRAM $30 \mathrm{~m}$ telescope at $88.8 \mathrm{GHz}$.

Table 1. Observed line parameters of $\mathrm{NCCNH}^{+}$in TMC-1 and L483.

\begin{tabular}{lcccc}
\hline \hline Transition & $\begin{array}{c}\text { Frequency } \\
(\mathrm{MHz})\end{array}$ & $\begin{array}{c}V_{\mathrm{LSR}} \\
\left(\mathrm{km} \mathrm{s}^{-1}\right)\end{array}$ & $\begin{array}{c}\Delta v \\
\left(\mathrm{~km} \mathrm{~s}^{-1}\right)\end{array}$ & $\begin{array}{c}\int T_{\mathrm{A}}^{*} \mathrm{~d} v \\
\left(\mathrm{~K} \mathrm{~km} \mathrm{~s}^{-1}\right)\end{array}$ \\
\hline$J=5-4$ & 44379.850 & $\begin{array}{c}\text { TMC-1 } \\
+5.68(8)\end{array}$ & $0.72(13)$ & $0.011(2)$ \\
$J=10-9$ & 88758.108 & $+5.91(9)$ & $0.48(12)$ & $0.006(1)$ \\
\hline \multicolumn{5}{c}{ L483 } \\
$J=5-4$ & 44379.850 & $+5.11(5)$ & $0.26(4)$ & $0.006(1)$ \\
$J=10-9$ & 88758.108 & $+5.31(9)$ & $0.49(13)$ & $0.017(4)$ \\
\hline
\end{tabular}

Notes. Numbers in parentheses are $1 \sigma$ uncertainties in units of the last digits.

\section{Results}

The emission lines observed toward TMC-1 and L483 and assigned to the $J=5-4$ and $J=10-9$ transitions of protonated cyanogen are shown in Fig. 1. Line parameters derived from Gaussian fits using GILDAS are listed in Table 1. The observations indicate that in TMC- 1 the $J=5-4$ line is more intense than the $J=10-9$, while in L483 the contrary is found. This fact suggests that $\mathrm{NCCNH}^{+}$probably has a higher rotational temperature and/or a more compact spatial distribution in L483 than in TMC- 1 . The $J=5-4$ and $J=10-9$ transitions have upper level energies of $6.4 \mathrm{~K}$ and $23.4 \mathrm{~K}$, respectively, and are observed with main beam sizes of 42 '. 6 and 27 '. 3 , respectively. 
Since we do not have information on the spatial distribution of $\mathrm{NCCNH}^{+}$in these two sources, we may assume that the emission size fills the main beam of the IRAM $30 \mathrm{~m}$ and Yebes $40 \mathrm{~m}$ telescopes to derive a first-order estimate of the column densities of protonated cyanogen. In TMC-1, we derive a beam-averaged column density of $(8.6 \pm 4.4) \times 10^{10} \mathrm{~cm}^{-2}$ and a rotational temperature of $5.8 \pm 0.8 \mathrm{~K}$. In L483, we derive a somewhat lower column density, $(3.9 \pm 1.8) \times 10^{10} \mathrm{~cm}^{-2}$, and a higher rotational temperature, $13.4 \pm 4.5 \mathrm{~K}$, that does, however, have a high degree of uncertainty. If we adopt $\mathrm{H}_{2}$ column densities of $1 \times 10^{22} \mathrm{~cm}^{-2}$ in TMC-1 (Cernicharo \& Guélin 1987) and $3 \times 10^{22} \mathrm{~cm}^{-2}$ in L483 (Tafalla et al. 2000; see also Agúndez et al. 2015), we end up with fairly low fractional abundances relative to $\mathrm{H}_{2}, 8.6 \times 10^{-12}$ in TMC-1 and $1.3 \times 10^{-12}$ in L483.

\section{Discussion}

The detection of $\mathrm{NCCNH}^{+}$is a good indication of the presence of NCCN in dark clouds. However, to have an idea of how abundant cyanogen is in this type of source, it is necessary to have a look at the chemistry of protonated molecules in general, and $\mathrm{NCCNH}^{+}$in particular. In a simplified chemical scheme, a protonated molecule $\mathrm{MH}^{+}$can be formed by proton transfer to $\mathrm{M}$ :

$$
\mathrm{XH}^{+}+\mathrm{M} \rightarrow \mathrm{MH}^{+}+\mathrm{X},
$$

where $\mathrm{M}$ must have a higher proton affinity than $\mathrm{X}$ in order to make the reaction exothermic. On the other hand, in cold dark clouds molecular cations are usually depleted by dissociative recombination with electrons

$\mathrm{MH}^{+}+\mathrm{e}^{-} \rightarrow$ products,

where the products can be assorted neutral fragments. Within this simple chemical scheme, at steady state we have

$$
\frac{\left[\mathrm{MH}^{+}\right]}{[\mathrm{M}]}=\frac{k_{\mathrm{PT}}}{k_{\mathrm{DR}}} \frac{\left[\mathrm{XH}^{+}\right]}{\left[\mathrm{e}^{-}\right]},
$$

where $k_{\mathrm{PT}}$ and $k_{\mathrm{DR}}$ are the rate constants of the reactions of proton transfer and dissociative recombination, respectively. Equation (3) suggests that high proton affinities of $\mathrm{M}$ would tend to enhance the rate of proton transfer (by increasing the number of available proton donors $\mathrm{XH}^{+}$and probably the associated rate constants $k_{\mathrm{PT}}$, hence the abundance ratio $\left[\mathrm{MH}^{+}\right] /[\mathrm{M}]$. The above chemical scheme may, however, not be suitable for all protonated molecules, making it necessary to build a chemical model that includes all relevant reactions to provide more precise predictions.

We built a pseudo time-dependent gas-phase chemical model of a dark cloud by adopting standard physical parameters $\left(T_{k}=\right.$ $\left.10 \mathrm{~K}, n_{\mathrm{H}}=2 \times 10^{4} \mathrm{~cm}^{-3}, \zeta=1.3 \times 10^{-17} \mathrm{~s}^{-1}, A_{V}=30\right)$ and "low metal" elemental abundances (see Agúndez \& Wakelam 2013). We adopted the UMIST RATE12 reaction network (McElroy et al. 2013) with a subset of reactions involving HCCN from Loison et al. (2015). We find that the chemical model validates the leading role of dissociative recombination, reaction (2), as the major destruction process of protonated molecules, except for $\mathrm{N}_{2} \mathrm{H}^{+}$and $\mathrm{HCO}_{2}^{+}$, which are also depleted to a large extent by proton transfer to $\mathrm{CO}$. We also find that, depending on whether the proton affinity of $\mathrm{M}$ is below or above that of $\mathrm{CO}$, the main proton donor $\mathrm{XH}^{+}$in reaction (1) is either $\mathrm{H}_{3}^{+}$or $\mathrm{HCO}^{+}$, respectively. However, some protonated molecules, such as $\mathrm{HCO}_{2}^{+}$, $\mathrm{HCS}^{+}$, and $\mathrm{HC}_{2} \mathrm{~S}^{+}$, are mainly formed by ion-neutral processes other than reaction (1), in which case Eq. (3) underestimates the abundance ratio $\left[\mathrm{MH}^{+}\right] /[\mathrm{M}]$.

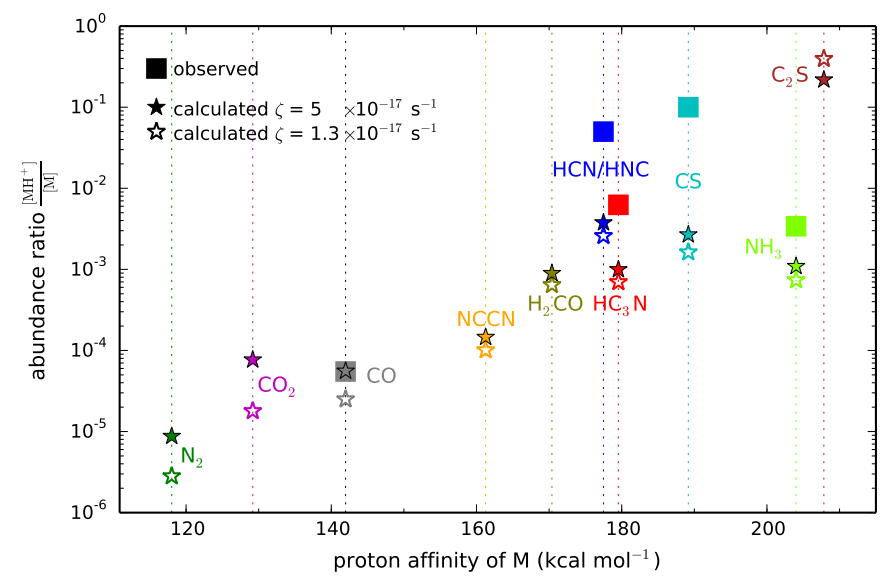

Fig. 2. Abundance ratios $\left[\mathrm{MH}^{+}\right] /[\mathrm{M}]$ between the protonated and nonprotonated form of some molecules as a function of the proton affinity of M. Calculated values at steady state (reached after some $10^{5} \mathrm{yr}$; see Fig. 3), adopting two different cosmic-ray ionization rates are compared with values derived from observations of TMC-1 (Agúndez \& Wakelam 2013). In the case of $\mathrm{NH}_{4}^{+}$, the observed value is derived from observations of the monodeuterated species in Barnard 1 (Cernicharo et al. 2013). The values for $\mathrm{HCN} / \mathrm{HNC}$ include both isomers and are plotted at the mean value of the proton affinities of HCN and HNC, $170.4 \mathrm{kcal} \mathrm{mol}^{-1}$ and $184.6 \mathrm{kcal} \mathrm{mol}^{-1}$, respectively.

Apart from $\mathrm{NCCNH}^{+}$and the widespread ions $\mathrm{HCO}^{+}$and $\mathrm{N}_{2} \mathrm{H}^{+}$, a few other protonated molecules have been observed in cold dark clouds: $\mathrm{HCS}^{+}$(Thaddeus et al. 1981), $\mathrm{HCNH}^{+}$ (Schilke et al. 1991), $\mathrm{HC}_{3} \mathrm{NH}^{+}$(Kawaguchi et al. 1994), $\mathrm{HCO}_{2}^{+}$ (Turner et al. 1999; Sakai et al. 2008), and $\mathrm{NH}_{3} \mathrm{D}^{+}$(Cernicharo et al. 2013). The abundance ratio $\left[\mathrm{MH}^{+}\right] /[\mathrm{M}]$ is reproduced well by the chemical model for $\mathrm{M}=\mathrm{CO}$ and $\mathrm{NH}_{3}$, although it is underestimated for $\mathrm{M}=\mathrm{HCN} / \mathrm{HNC}, \mathrm{HC}_{3} \mathrm{~N}$, and especially $\mathrm{CS}$ (see Fig. 2). Taking into account that $\mathrm{HCNH}^{+}, \mathrm{HC}_{3} \mathrm{NH}^{+}$, and $\mathrm{HCS}^{+}$are mainly destroyed by dissociative recombination with electrons, whose rate constants are well known from experiments (Semaniak et al. 2001; Geppert et al. 2004; Montaigne et al. 2005), and that the rate constants of formation by proton transfer from $\mathrm{HCO}^{+}$are usually well constrained experimentally (Anicich 2003), it is likely that the underestimation occurs because the chemical model misses important formation routes to $\mathrm{HCNH}^{+}, \mathrm{HC}_{3} \mathrm{NH}^{+}$, and $\mathrm{HCS}^{+}$.

The abundance ratios $\left[\mathrm{MH}^{+}\right] /[\mathrm{M}]$ are sensitive to the degree of ionization and thus to various physical parameters, such as the cosmic-ray ionization rate $\zeta$ (the sensitivity is marked more for low proton affinities; see Fig. 2) and the volume density of particles. The higher the density, the lower the ionization fraction and thus the lower the importance of protonated molecules. It is interesting to note that both the chemical model and the observations suggest a trend in which the abundance ratio $\left[\mathrm{MH}^{+}\right] /[\mathrm{M}]$ increases with the increasing proton affinity of $\mathrm{M}$. Since the destruction of $\mathrm{MH}^{+}$is controlled by dissociative recombination with electrons, whose rate constants are usually similar within one order of magnitude, the trend can be explained in terms of an enhanced formation rate of $\mathrm{MH}^{+}$with the increasing proton affinity of $\mathrm{M}$, resulting from the appearance of multiple formation pathways to $\mathrm{MH}^{+}$through exothermic ion-neutral reactions of proton transfer or another type. We may therefore expect to find other abundant protonated molecules $\mathrm{MH}^{+}$in dark clouds, as long as $\mathrm{M}$ has a high proton affinity and a sufficiently high abundance. Molecules, such as $\mathrm{H}_{2} \mathrm{COH}^{+}$(previously detected by Ohishi et al. 1996 toward warm, but not cold, clouds), $\mathrm{OCSH}^{+}$, 
A\&A 579, L10 (2015)

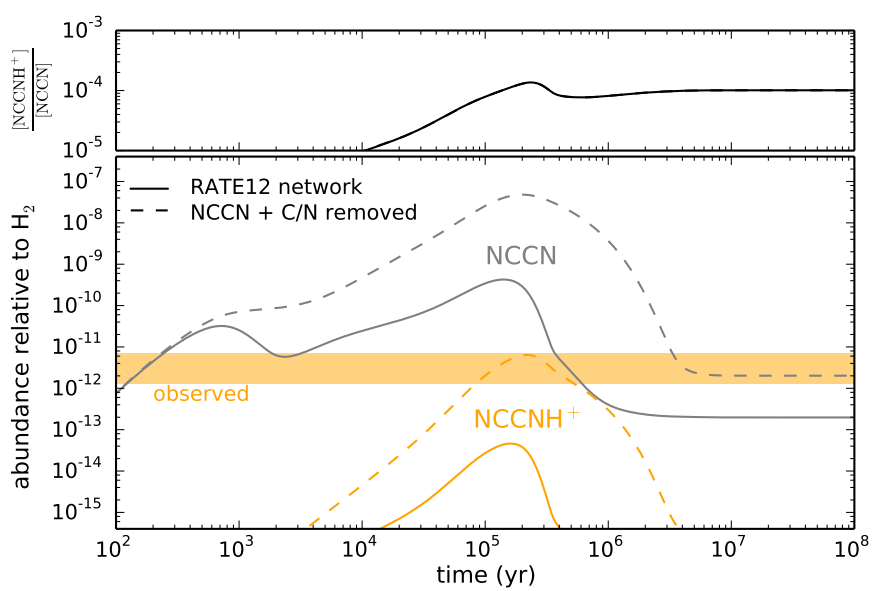

Fig. 3. Calculated abundances of $\mathrm{NCCNH}^{+}$and $\mathrm{NCCN}$ as a function of time using the UMIST RATE12 chemical network (solid lines) and after removing the reactions of destruction of NCCN with $\mathrm{C}$ and $\mathrm{N}$ atoms (dashed lines), in both cases adopting $\zeta=1.3 \times 10^{-17} \mathrm{~s}^{-1}$. The range of abundances of $\mathrm{NCCNH}^{+}$observed in TMC-1 and $\mathrm{L} 483$ is also indicated. The calculated abundance ratio $\left[\mathrm{NCCNH}^{+}\right] /[\mathrm{NCCN}]$ is shown in the upper panel as a function of time.

and $\mathrm{C}_{2} \mathrm{H}_{3}^{+}$, are potentially detectable. However, the most promising candidate is probably $\mathrm{HC}_{2} \mathrm{~S}^{+}$, given that it has a high proton affinity and dipole moment (Barrientos \& Largo 1991; Maclagan \& Sudkeaw 1992; Puzzarini 2008) and it is predicted to be just a few times less abundant than $\mathrm{C}_{2} \mathrm{~S}$, whose column density in dark clouds is in the range $(1-100) \times 10^{12} \mathrm{~cm}^{-2}$ (Fuente et al. 1990; Suzuki et al. 1992).

The chemical model predicts a low abundance of $\mathrm{NCCNH}^{+}$ (see Fig. 3), although it is not clear whether it is because the model underestimates the abundance of NCCN or the abundance ratio $\left[\mathrm{NCCNH}^{+}\right] /[\mathrm{NCCN}]$. According to the chemical model, $\mathrm{NCCNH}^{+}$is formed by proton transfer from $\mathrm{HCO}^{+}$to $\mathrm{NCCN}$ and destroyed by dissociative recombination with electrons, the rate constants of which are not known, although they are unlikely to be radically different from the values guessed in the UMIST RATE12 reaction network. We thus expect the abundance ratio $\left[\mathrm{NCCNH}^{+}\right] /[\mathrm{NCCN}]$ to be around $10^{-4}$, as predicted by the chemical model, or higher, if important formation routes to $\mathrm{NCCNH}^{+}$are missing in the model. If the abundance ratio $\left[\mathrm{NCCNH}^{+}\right] /[\mathrm{NCCN}]$ is correctly predicted, then the low fractional abundance predicted for $\mathrm{NCCNH}^{+}$must arise from an underestimation of the abundance of NCCN. In the chemical model, cyanogen is essentially formed by the reaction

$\mathrm{HNC}+\mathrm{CN} \rightarrow \mathrm{NCCN}+\mathrm{H}$,

for which Petrie et al. (2003) estimate a rate constant of $2 \times 10^{-10} \mathrm{~cm}^{3} \mathrm{~s}^{-1}$, and depleted by reaction with $\mathrm{C}$ and $\mathrm{N}$ atoms, whose rate constants at room temperature are inferred to be a few $10^{-11} \mathrm{~cm}^{3} \mathrm{~s}^{-1}$ (Whyte \& Phillips 1983; Safrany \& Jaster 1968). The calculated abundance of $\mathrm{NCCN}$ (and $\mathrm{NCCNH}^{+}$) is very sensitive to these rate constants (see Fig. 3). Therefore, a better understanding of the low temperature chemical kinetics of reaction (4) and the reactions of NCCN with $\mathrm{C}$ and $\mathrm{N}$ atoms seem the most immediate step toward better constraining the chemistry of cyanogen in cold interstellar clouds. Additional uncertainties could come from other reactions that affect the precursors HNC and $\mathrm{CN}$. For example, the systematic use of enhanced rate constants for most ion-polar reactions, adopted in the UMIST RATE12 network, results in lower abundances for HNC, NCCN, and $\mathrm{NCCNH}^{+}$, as compared with the use of a chemical network in which these rate constant enhancements are not adopted (see, e.g., Woodall et al. 2007). Formation of cyanogen on the surface of dust grains followed by some non-thermal desorption process could also be explored.

If we trust the abundance ratio $\left[\mathrm{NCCNH}^{+}\right] /[\mathrm{NCCN}]$ of $\sim 10^{-4}$ calculated by the chemical model, the abundance of $\mathrm{NCCN}$ in dark clouds could be as high as $(1-10) \times 10^{-8}$ relative to $\mathrm{H}_{2}$, so comparable to that of other abundant nitriles, such as $\mathrm{HCN}, \mathrm{HNC}$, and $\mathrm{HC}_{3} \mathrm{~N}$.

Acknowledgements. We thank the IRAM $30 \mathrm{~m}$ staff for their help during the observations and our referee, John Black, for useful comments. M.A. and J.C. acknowledge funding support from the European Research Council (ERC Grant 610256: NANOCOSMOS) and from Spanish MINECO through grants CSD2009-00038, AYA2009-07304, and AYA2012-32032. Authors from Yebes Observatory acknowledge funding by MINECO through grant FIS2012-32096. E.R. and M.G. acknowledge funding support from the CNRS program "Physique et Chimie du Milieu Interstellaire" (PCMI).

\section{References}

Agúndez, M., \& Wakelam, V. 2013, Chem. Rev., 113, 8710

Agúndez, M., Cernicharo, J., Guélin, M., et al. 2008, A\&A, 478, L19

Agúndez, M., Cernicharo, J., \& Guélin, M. 2014, A\&A, 570, A45

Agúndez, M., Cernicharo, J., \& Guélin, M. 2015, A\&A, 577, L5

Amano, T., \& Scappini, F. 1991, J. Chem. Phys., 95, 2280

Anicich, V. G. 2003, JPL Publication 03-19

Barrientos, C., \& Largo, A. 1991, Chem. Phys. Lett., 184, 168

Bell, M. B., Feldman, P. A., Travers, M. J., et al. 1997, ApJ, 483, L61

Botschwina, P., \& Sebald, P. 1990, Chem. Phys., 141, 311

Broten, N. W., Oka, T., Avery, L. W., et al. 1978, ApJ, 223, L105

Cernicharo, J. 1985, IRAM Report, 52

Cernicharo, J., \& Guélin, M. 1987, A\&A, 176, 299

Cernicharo, J., Marcelino, N., Roueff, E., et al. 2012, ApJ, 759, L43

Cernicharo, J., Tercero, B., Fuente, A., et al. 2013, ApJ, 771, L10

Coustenis, A., Bézard, B., Gautier, D., et al. 1991, Icarus, 89, 152

Fray, N., Bénilan, Y., Cottin, H., Gazeau, M.-C., \& Crovisier, J. 2005, Planet. Space Sci., 53, 1243

Fuente, A., Cernicharo, J., Barcia, A., \& Gómez-González, J. 1990, A\&A, 231, 151

Geppert, W. D., Ehlerding, A., Hellberg, F., et al. 2004, ApJ, 613, 1302

Gottlieb, C. A., Apponi, A. J., McCarthy, M. C., \& Thaddeus, P. 2000, J. Chem. Phys., 113, 1910

Jolly, A., Cottini, V., Fayt, A. et al. 2015, Icarus, 248, 340

Kawaguchi, K., Kasai, Y., Ishikawa, S.-I., et al. 1994, ApJ, 420, L95

Kołos, R., \& Grabowski, Z. R. 2000, Ap\&SS, 271, 65

Kunde, V. G., Aikin, A. C., Hanel, R. A., et al. 1981, Nature, 292, 686

Loison, J. C., Hébrard, E., Dobrijevic, M., et al. 2015, Icarus, 247, 218

Maclagan, R. G. A. R., \& Sudkeaw, P. 1992, Chem. Phys. Lett., 194, 147

Marcelino, N., Cernicharo, J., Roueff, E., et al. 2005, ApJ, 620, 308

Marcelino, N., Cernicharo, J., Agúndez, M., et al. 2007, ApJ, 665, L127

Marcelino, N., Cernicharo, J., Tercero, B., \& Roueff, E. 2009, ApJ, 690, L27

McElroy, D., Walsh, C., Markwick, A. J., et al. 2013, A\&A, 550, A36

Montaigne, H., Geppert, W. D., Semaniak, J., et al. 2005, ApJ, 631, 653

Ohishi, M., Ishikawa, S.-I., Amano, T., et al. 1996, ApJ, 471, L61

Pardo, J. R., Cernicharo, J., \& Serabyn, G. 2001, IEEE Trans. Antennas Propag., 49, 1683

Pardo, J. R., Cernicharo, J., \& Goicoechea, J. R. 2005, ApJ, 628, 275

Petrie, S., Millar, T. J., \& Markwick, A. J. 2003, MNRAS, 341, 609

Puzzarini, C. 2008, Chem. Phys., 346, 45

Safrany, D. R., \& Jaster, W. 1968, J. Phys. Chem., 72, 3305

Sakai, N., Sakai, T., Aikawa, Y., \& Yamamoto, S. 2008, ApJ, 675, L89

Schilke, P., Walmsley, C. M., Millar, T. J., \& Henkel, C. 1991, A\&A, 247, 487

Semaniak, J., Minaev, B. F., Derkatch, A. M., et al. 2001, ApJS, 135, 275

Suzuki, H., Yamamoto, S., Ohishi, M., et al. 1992, ApJ, 392, 551

Tafalla, M., Myers, P. C., Mardones, D., \& Bachiller, R. 2000, A\&A, 359, 967

Thaddeus, P., Guélin, M., \& Linke, R. A. 1981, ApJ, 246, L41

Turner, B. E., Terzieva, R., \& Herbst, E. 1999, ApJ, 518, 699

Whyte, A. R., \& Phillips, L. F. 1983, Chem. Phys. Lett., 98, 590

Winnewisser, G., \& Walmsley, C. M. 1978, A\&A, 70, L37

Woodall, J., Agúndez, M., Markwick-Kemper, A. J., \& Millar, T. J. 2007, A\&A, 466, 1197 